\title{
Complete response induced by concurrent chemoradiotherapy in a patient with NUT carcinoma: a case report
}

\section{Joji Muramatsu}

Sapporo Medical University: Sapporo Ika Daigaku https://orcid.org/0000-0002-7711-5450

Kohichi Takada ( $\sim$ ktakada@sapmed.ac.jp )

Sapporo Medical University School of Medicine

\section{Shintaro Sugita}

Sapporo Medical University: Sapporo Ika Daigaku

\section{Takaaki Tsuchiya}

Sapporo Medical University: Sapporo Ika Daigaku

\section{Keisuke Yamamoto}

Sapporo Medical University: Sapporo Ika Daigaku

\section{Kazuyuki Murase}

Sapporo Medical University: Sapporo Ika Daigaku

\section{Saki Ameda}

Sapporo Medical University: Sapporo Ika Daigaku

\section{Yohei Arihara}

Sapporo Medical University: Sapporo Ika Daigaku

\section{Koji Miyanishi}

Sapporo Medical University: Sapporo Ika Daigaku

\section{Koh-Ichi Sakata}

Sapporo Medical University: Sapporo Ika Daigaku Junji Kato

Sapporo Medical University: Sapporo Ika Daigaku

\section{Case Report}

Keywords: NUT carcinoma, concurrent chemoradiotherapy, VDC-IE regimen

Posted Date: February 9th, 2021

DOI: https://doi.org/10.21203/rs.3.rs-202205/v1 
License: (c) (i) This work is licensed under a Creative Commons Attribution 4.0 International License. Read Full License 


\section{Abstract}

\section{Background}

NUT carcinoma (NC) is a rare and extremely aggressive carcinoma that occurs in young individuals. Although NC has a dismal prognosis, recommended therapies have not been established.

\section{Case presentation}

An 18-year-old man presented with sudden vision loss in his left eye. Magnetic resonance imaging revealed a rapidly growing tumor, which had directly invaded the left optic nerve and epidural space, originating from the left posterior ethmoid sinus. A pathological examination of the tumor revealed a dense proliferation of polygonal-to-round tumor cells with enlarged round nuclei and conspicuous nucleoli, and positive staining for NUT protein in the nuclei of tumor cells. The patient was diagnosed with locally advanced NC. We initiated concurrent chemoradiotherapy (CCRT), consisting of chemotherapy with vincristine, doxorubicin, and cyclophosphamide (VDC), alternating with ifosphamide and etoposide (IE) according to a regimen for Ewing sarcoma, plus radiation therapy (70 Gy/35 Fr). The patient completed CCRT and achieved a complete response.

Conclusions

CCRT, comprising a VDC-IE regimen, can be a useful treatment option for adolescent and young adult patients with $\mathrm{NC}$, even when the cancer is at a locally advanced unresectable stage.

\section{Background}

NUT carcinoma (NC), which usually involves midline body parts such as the head, neck and thorax, is a rare and highly aggressive carcinoma that occurs in young individuals. NC consists mainly of sheets of undifferentiated cells with focal abrupt dyskeratosis and squamous differentiation. This tumor entity is defined by an acquired chromosomal rearrangement of the NUT gene at the 15q14 locus. The diagnosis requires identifying the chromosomal rearrangement of NUT with fluorescence in situ hybridization (FISH), reverse transcription polymerase chain reaction, or next-generation sequencing [1]. Furthermore, a specific monoclonal antibody against NUT has been frequently used for the diagnosis of NC, showing a specificity of $100 \%$ and sensitivity of $87 \%$ [2]. Although NC has a dismal prognosis, recommended therapies have not been established, especially in patients who are ineligible for surgical interventions. Herein, we describe the successful treatment of an adolescent and young adult (AYA) patient with unresectable NC using concurrent chemoradiotherapy (CCRT).

\section{Case Presentation}

An 18-year-old man presented with sudden vision loss in his left eye. He was initially diagnosed with left retrobulbar neuritis and treated with steroid-pulse therapy for three days by an ophthalmologist. However, 
his left vision did not improve. Magnetic resonance imaging (MRI) revealed a tumor in the left posterior ethmoid sinus that had directly invaded the left optic nerve and epidural space (Fig. 1A). To manage the tumor, the patient was referred to the Department of Otolaryngology and Medical Oncology at our hospital. Laboratory examinations showed a slightly elevated level of squamous cell carcinoma antigen (1.8 ng/mL [normal range: $0-1.5 \mathrm{ng} / \mathrm{mL}$ ]). Positron emission tomography (PET)-computed tomography (CT) demonstrated a left ethmoid sinus mass with increased fluorodeoxyglucose uptake but evidence of metastasis was not found (Fig. 1B). The tumor rapidly grew during examinations including in images. Endoscopic-assisted surgery was performed to diagnose the tumor; we found that the tumor had filled the area behind the basal lamella of the middle turbinate, and directly invaded the left optic nerve and epidural space (Fig. 2). A pathological examination of the tumor revealed a dense proliferation of polygonal-to-round tumor cells with enlarged round nuclei and conspicuous nucleoli (Fig. 3A). Immunohistochemical analyses identified positive staining for NUT protein in the nuclei of tumor cells (Fig. 3B). The malignant cells retained nuclear staining for INI-1, and were positive for CK-AE1/AE3 (focal), CK5/6 (mild), p40 (focal), and vimentin. We performed FISH analysis of NUTM1-BRD4 to detect the rearrangement. However, NUTM-1BRD4 rearrangement was not detected. In contrast, FISH analysis of NUTM1 showed a NUTM1 split signal in $90 \%$ of the tumor (Fig. 4). The patient was diagnosed with locally advanced unresectable NC. We initiated CCRT, consisting of chemotherapy with VDC (vincristine at a dose of $1.5 \mathrm{mg} / \mathrm{sqm}$ on day 1 , doxorubicin at a dose of $75 \mathrm{mg} / \mathrm{sqm}$ on days $1-2$, and cyclophosphamide at a dose of $1200 \mathrm{mg} / \mathrm{sqm}$ on day 1), alternating with IE (ifosphamide at a dose of $1.8 \mathrm{~g} / \mathrm{sqm}$ on days $1-5$ and etoposide at a dose of $100 \mathrm{mg} / \mathrm{sqm}$ on days $1-5$ ) repeating every 14 days, according to a regimen for Ewing sarcoma [3], plus radiation therapy (70 Gy/35 Fr). To avoid the cardiotoxicity of doxorubicin, we administered the VDC regimen for up to five cycles, subsequently replacing this with a VAC-(consisting of vincristine, actinomycin $D$ [at a dose of $1.25 \mathrm{mg} / \mathrm{sqm}$ on day 1]) IE regimen, which was continued for four cycles. Conventional radiation therapy was applied to the tumor simultaneously from two weeks before the first VDC treatment. The patient was treated with $2.0 \mathrm{~Gy}$ once daily, with a total number of 20 fractions delivering a cumulative dose of $40 \mathrm{~Gy}$ to the tumor. Thereafter, intensity modulated radiotherapy was continued with 2.0 Gy once daily up to a cumulative dose of 30 Gy to control the tumor completely. Except for Grade 3 febrile neutropenia, no other serious adverse events have been observed. After completion of the CCRT, we conducted MRI, and PET-CT (Fig. 5), and biopsied the tumor area. Images and pathological examination revealed no evidence of residual tumor, and we therefore diagnosed the patient as having achieved a complete response (CR). At the time of writing, the patient had maintained a CR for 8 months after the cessation of treatment. The patient has now been alive for 18 months from the initial diagnosis, although his left vision has not recovered.

\section{Discussion And Conclusions}

A recent retrospective study demonstrated that median overall survival (OS) was 6.5 months in patients with NC, with around a $70 \%$ chance of death within a year, highlighting the need to develop effective treatment strategies for this disease [3]. Currently, most patients with $\mathrm{NC}$ receive multimodal treatment composed of a combination of surgery, chemotherapy, and radiation. In patients with NC of the head and 
neck, aggressive surgery with or without post-operative chemo-radiotherapy or radiation are recommended because the treatment is associated with good clinical outcomes [5]. Of note, upfront complete resection prolonged OS significantly. However, in our case, we conducted CCRT consisting of a VDC-IE regimen since the patient was ineligible for a complete resection as the tumor had directly invaded the epidural space; the patient also wanted to retain his physical appearance. We selected CCRT comprised of a VDC-IE regime as a first-line treatment because several reports have demonstrated that such a regimen was effective for Ewing sarcoma; combined with radiotherapy and surgery this regimen was also effective for patients with NC (Table). To maximize the dose intensity, we treated the patient every two weeks [11]. According to a previous report and our case, regimens for Ewing sarcoma are also adequate and useful for not only children but also AYA generations as patients with NC [9]. Unfortunately, other chemotherapeutic regimens have not been successful clinically $[5,12]$. Therefore, to date, regimens for Ewing sarcoma are the best strategies for patients with advanced-stage NC or in a CCRT or adjuvant chemotherapy setting.

Interestingly, most patients with sustained $\mathrm{CR}$ received upfront or planned surgery (Table 1), implying surgery was effective in the treatment of patients with NC. In our case, a surgical resection of the tumor was not performed, and, as such, we need to be attentive to any local recurrence.

Recently, several bromodomain and extra-terminal (BET) protein inhibitors have induced clinical responses in patients with $\mathrm{NC}[13,14]$. Consequently, BET inhibitors will continue to be used for patients with NC, and to improve the prognosis in the near future.

Our findings suggest CCRT, comprising a VDC-IE regimen, can be a useful treatment option for AYA patients with $\mathrm{NC}$, even when the cancer is at a locally advanced unresectable stage.

\section{Declarations}

\section{Ethics Approval and Consent to Participate}

Not applicable

\section{Consent for Publication}

Written informed consent was obtained from the patient for publication.

\section{Availability of data and materials}

Not applicable

\section{Competing interests}

The authors declare that they have no competing interests.

\section{Funding}


Not applicable

\section{Author's contributions}

$\mathrm{JM}$ and KT: analyzed data, and wrote and edited the manuscript. SS: performed the pathological analysis, including FISH. TT and KS: were radiologists who provided radiation therapy. KY: performed the nasopharyngeal endoscopy and tumor biopsy. KM, SA, KM, JK: was involved in the patient's care. YA: advised on the pathophysiology of NC. All authors have read and approved the final manuscript.

\section{Acknowledgements}

All authors would like to thank the patient and his family for allowing the publication of this case study.

\section{References}

1. Naiquan M, Zhiling L, Junwei W,Kai L, Shoufeng W, Shaomian Q, et al. Diagnosis of NUT carcinoma of lung origin by next-generation sequencing: case report and review of the literature. Cancer Biol Ther. 2019;20(2):150-6.

2. Herbert H, Laura AJ, Christopher JF, Katherine C, Roberto DP, Edward BS, et al. Diagnosis of NUT midline carcinoma using a NUT-specific monoclonal antibody. Am J Surg Pathol. 2009;33(7):984-91.

3. Chin M, Yokoyama R, Sumi M, Okita H, Kawai A, Hosono A, et al. Multimodal treatment including standard chemotherapy with vincristine, doxorubicin, cyclophosphamide, ifosfamide, and etoposide for the Ewing sarcoma family of tumors in Japan: results of the Japan Ewing Sarcoma Study 04. Pediatr Blood Cancer. 2020;67:e28194.

4. Chau NG, Clement M, Danga K, Al-Sayegh H, Nardi V, Barrette R, et al. An Anatomical Site and Genetic-Based Prognostic Model for Patients With Nuclear Protein in Testis (NUT) Midline Carcinoma: Analysis of 124 Patients. JNCI Cancer Spectr. 2019;4(2):pkz094.

5. Chau NG, Hurwitz S, Mitchell CM, Aserlind A, Grunfeld N, Kaplan L, et al. Intensive treatment and survival outcomes in NUT midline carcinoma of the head and neck. Cancer. 2016;122(23):3632-40.

6. Mertens F, Wiebe T, Adlercreutz C, Mandahl N, French CA. Successful treatment of a child with $\mathrm{t}(15 ; 19)$ - positive tumor. Pediatr Blood Cancer. 2007;49(7):1015-7.

7. Storck S, Kennedy AL, Marcus KJ, Teot L, Vaughn J, Gnekow AK, et al. Pediatric NUT-midline carcinoma: Therapeutic success employing a sarcoma based multimodal approach. Pediatr Hematol Oncol. 2017; 34(4):231-7.

8. Arimizu K, Hirano G, Makiyama C, Matsuo M, Sasaguri T, Makiyama A. NUT carcinoma of the nasal cavity that responded to a chemotherapy regimen for Ewing's sarcoma family of tumors: a case report. BMC Cancer. 2018;18:1134.

9. Leeman R, Pinkney K, Bradley JA, Ruiz R, DuBois SG, French C, et al. NUT Carcinoma Without Upfront Surgical Resection: A Case Report. J Pediatr Hematol Oncol. 2020; doi:

10.1097/MPH.0000000000001865. 
10. Sopfe J, Greffe B, Treece AL. Metastatic NUT Midline Carcinoma Treated With Aggressive Neoadjuvant Chemotherapy, Radiation, and Resection: A Case Report and Review of the Literature. J Pediatr Hematol Oncol. 2021;43(1):e73-5.

11. Womer RB, West DC, Krailo MD, Dickman PS, Pawel BR, Grier HE, et al. Randomized Controlled Trial of Interval-Compressed Chemotherapy for the Treatment of Localized Ewing Sarcoma: A Report From the Children's Oncology Group. J Clin Oncol. 2012;30:4148-54.

12. Prasad M, Baheti A, Ramadwar M, Chinnaswamy G, Vora T, Qureshi S. Pediatric NUT Carcinoma Is a Rare and Challenging Tumor: Single Center Experience of Five Children. Oncologist. 2019;24(11):e1232-5.

13. Shapiro GI, LoRusso P, Dowlati A, Do KT, Jacobson CA, Vaishampayan U, et al. A Phase 1 study of R06870810, a novel bromodomain and extra-terminal protein inhibitor, in patients with NUT carcinoma, other solid tumours, or diffuse large B-cell lymphoma. Br J Cancer. 2020; doi: 10.1038/s41416-020-01180-1.

14. Lewin J, Soria JC, Stathis A, Delord JP, Peters S, Awada A, et al. Phase Ib Trial With Birabresib, a Small-Molecule Inhibitor of Bromodomain and Extraterminal Proteins, in Patients With Selected Advanced Solid Tumors. J Clin Oncol. 2018;36(30):3007-14.

\section{Tables}

Table Efficacies of regimens for Ewing sarcoma in patients with NUT carcinoma.

Table Muramatsu et al.

\begin{tabular}{|c|c|c|c|c|c|c|c|c|}
\hline Case & $\begin{array}{l}\text { Age (y) } \\
\text { /Sex }\end{array}$ & Primary & Metastasis & $\begin{array}{l}\text { NUT } \\
\text { fusion } \\
\text { type }\end{array}$ & $\begin{array}{l}1^{\text {st }} \text { line } \\
\text { therapy }\end{array}$ & $\begin{array}{l}2^{\text {nd }} \text { line } \\
\text { Therapy }\end{array}$ & Efficacy & os \\
\hline $1^{6}$ & $10 / \mathrm{M}$ & ilium & - & BRD4 & $\begin{array}{l}\text { VAI-PAI-VAl x4 } \\
\text { RT( } 60 \mathrm{~Gy} / 40 \mathrm{Fr})\end{array}$ & - & CR & $\begin{array}{c}13 \mathrm{y} \\
\text { (alive) }\end{array}$ \\
\hline $2^{7}$ & $9 / M$ & sublingual gland & cervical L/N & NA & $\begin{array}{c}\text { Surgery } \\
\text { VAI-PAI-VAI x4 } \\
\text { RT (54 Gy/32 Fr) }\end{array}$ & - & CR & $\begin{array}{c}6 y \\
\text { (alive) }\end{array}$ \\
\hline $3^{7}$ & $9 / M$ & parotid gland & cervical L/N & NA & $\begin{array}{c}\text { Surgery } \\
\text { VAl-PAI-VAI x4 } \\
\text { RT }(59.4 \mathrm{~Gy} / 33 \mathrm{Fr})\end{array}$ & - & CR & $\begin{array}{c}15 \mathrm{M} \\
\text { (alive) }\end{array}$ \\
\hline $4^{8}$ & $49 / \mathrm{M}$ & nasal sinuses & ilium & NA & VDCX 6 & $\begin{array}{c}\text { CDDP } \\
\text { RT (75 Gy/35 Fr) } \\
\text { Surgery }\end{array}$ & PD & $9 \mathrm{M}$ \\
\hline $5^{\circ}$ & $15 / F$ & nasal sinuses & - & BRD3 & $\begin{array}{c}\text { VAI-PAI-VAl x4 } \\
\text { RT(68.4 Gy/38 Fr) } \\
\text { Surgery }\end{array}$ & - & CR & $\begin{array}{c}34 \mathrm{M} \\
\text { (alive) }\end{array}$ \\
\hline $6^{10}$ & $12 / F$ & nasal sinuses & $\begin{array}{c}\text { vertebral body, } \\
\text { right sacrum, } \\
\text { femur, tibia }\end{array}$ & NA & $\begin{array}{c}\text { VDC-IE x14 } \\
\text { RT(55.8 Gy) } \\
\text { Surgery }\end{array}$ & - & CR & $\begin{array}{l}40 \mathrm{M} \\
\text { (alive) }\end{array}$ \\
\hline Our case & $18 / \mathrm{M}$ & ethmoid sinus & - & NA & $\begin{array}{c}\text { VDC-IE x17 } \\
\text { RT(70 Gy/35 Fr) }\end{array}$ & - & CR & $\begin{array}{c}18 \mathrm{M} \\
\text { (alive) }\end{array}$ \\
\hline
\end{tabular}

Cases 1-3, and 5 were treated with a vincristine, doxorubicin, ifosfamide (VAI) - cisplatin, doxorubicin, ifosfamide (PAI) regimen. Cases 4, 6, and our case were treated with a vincristine, doxorubicin, and cyclophosphamide (VDC) or VDC-ifosfamide and etoposide (IE) regimen. 
CR, complete response; PD, progressive disease; CDDP, cisplatin; L/N, lymph node; NA, not applicable; OS, overall survival; RT, radiotherapy

\section{Figures}
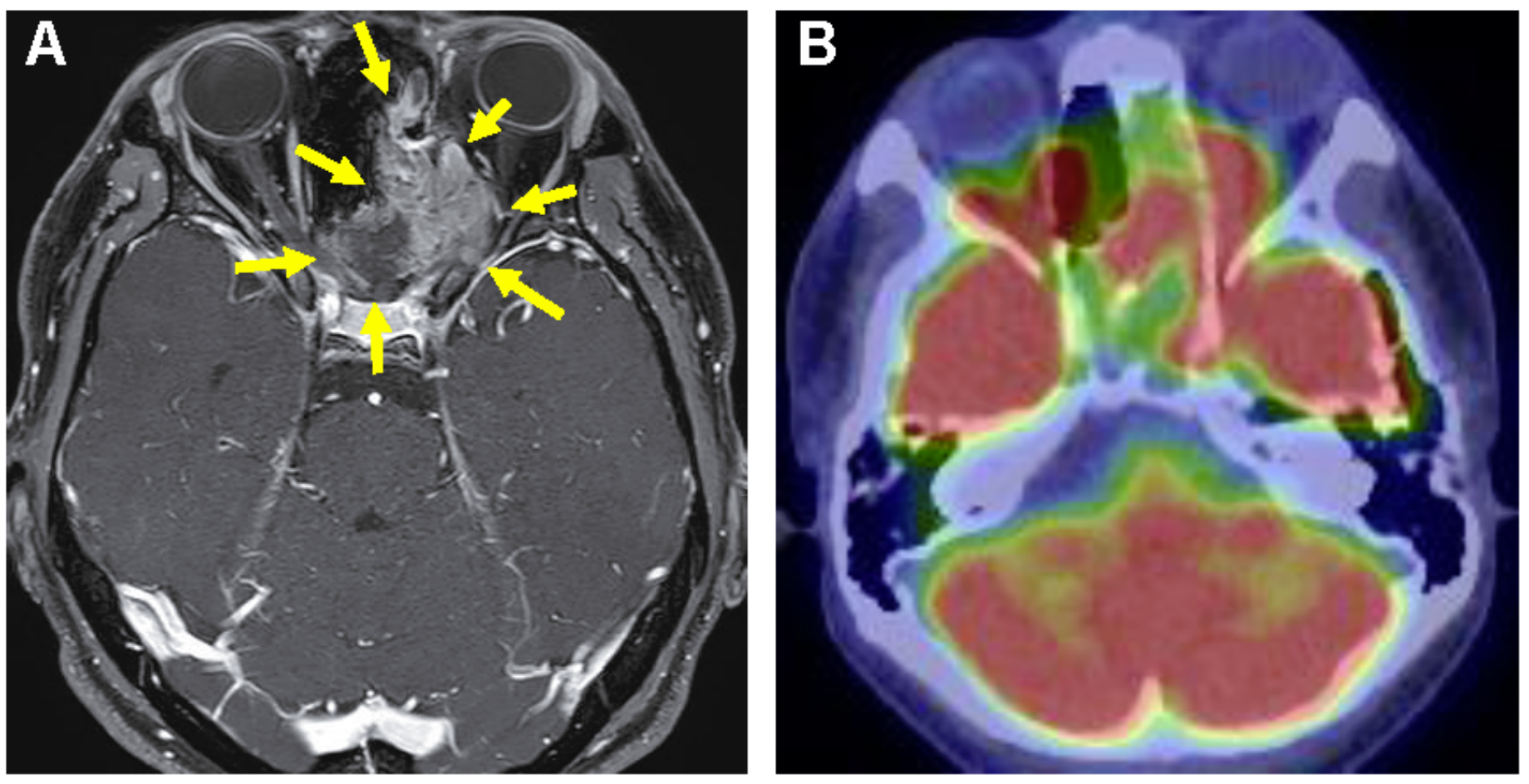

Figure 1

Magnetic resonance imaging (MRI) (A) and positron emission tomography (PET)-computed tomography (CT) scan (B). MRI revealed a left posterior ethmoid sinus mass (allows), and PET-CT demonstrated the mass with increased FDG uptake. 


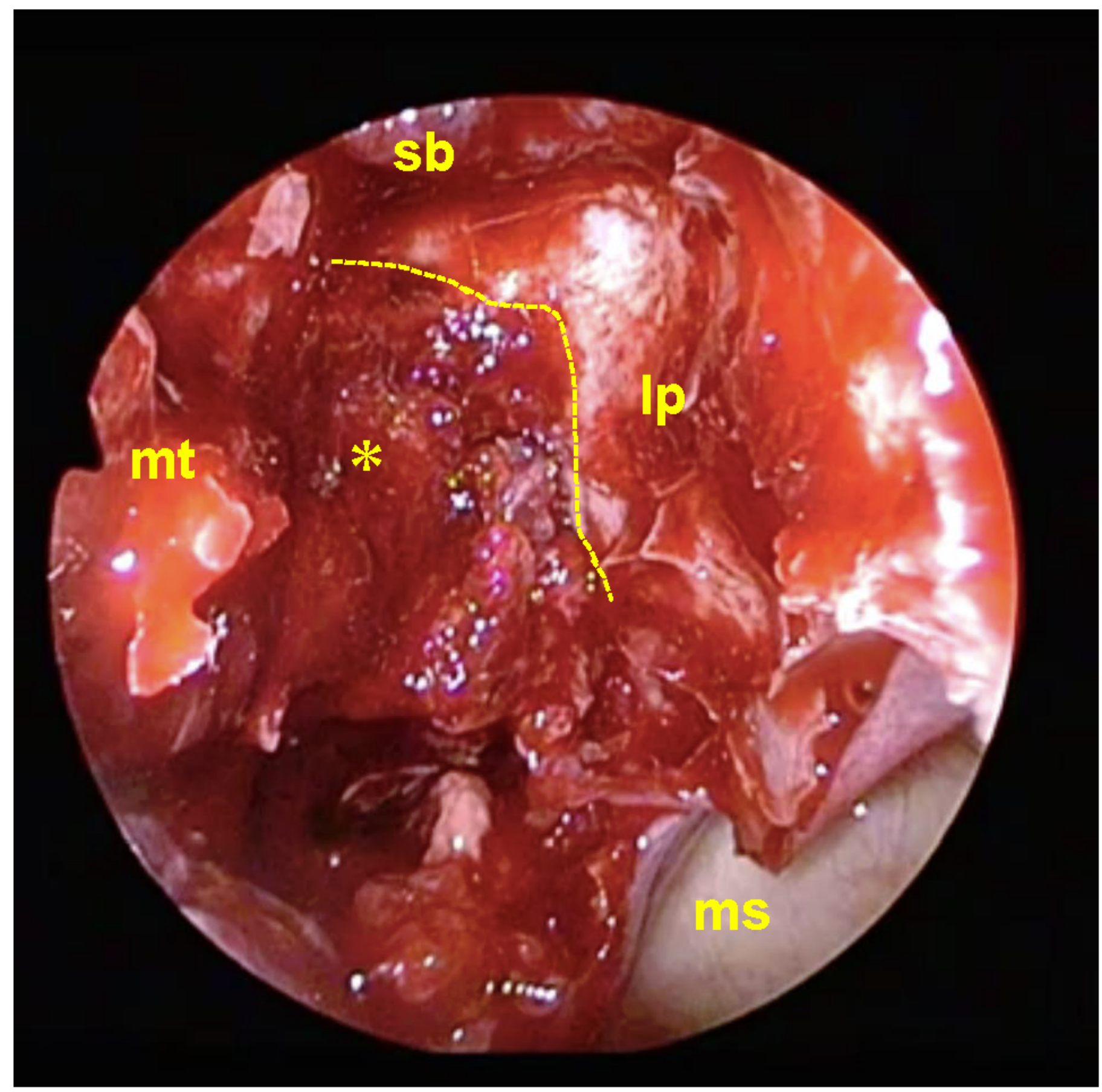

Figure 2

Endoscopic findings of the middle nasal meatus in an operation. The tumor extended from the posterior ethmoid sinus into the skull base and the orbit (dotted line). Tumor ( $(\mathbb{)})$, lamina papyracea (Ip), maxillary sinus (ms), middle turbinate (mt), skull base (sb). 


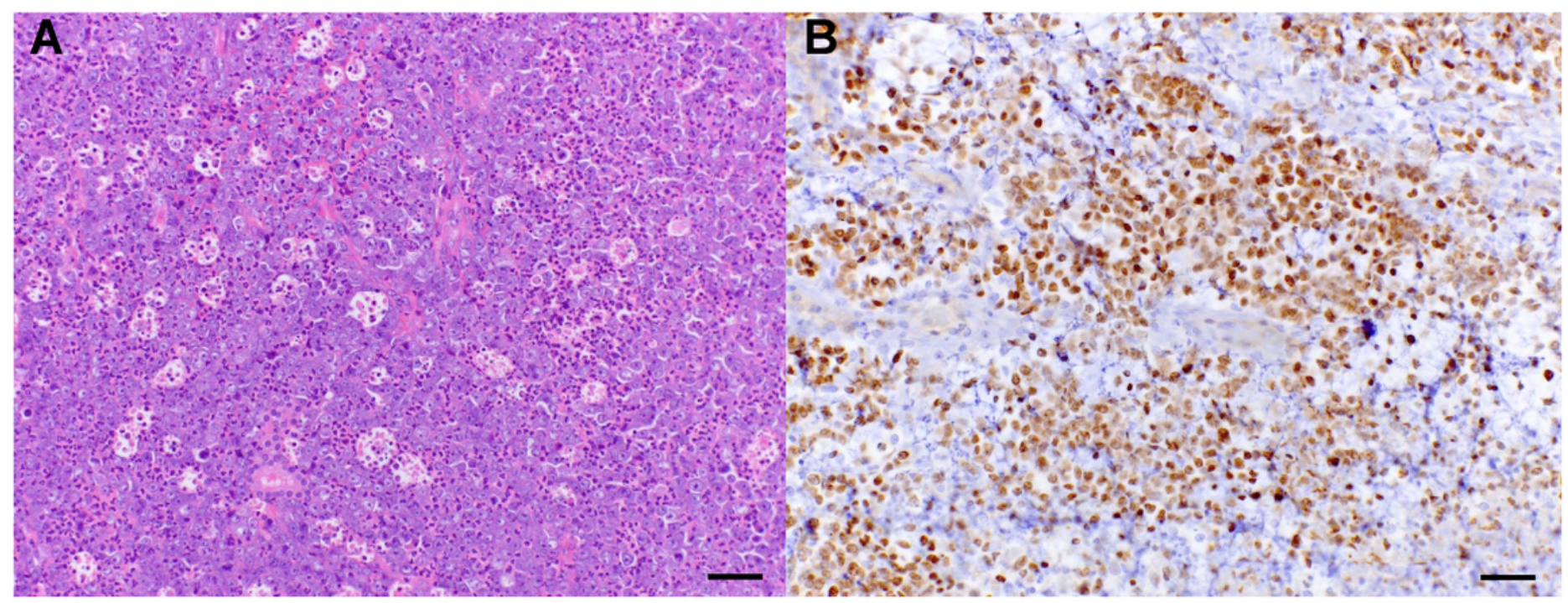

\section{Figure 3}

Microscopic findings of the resected tumor. (A) Hematoxylin and eosin (H\&E) staining. Scale bars, $50 \mu m$. (B) NUT staining. 


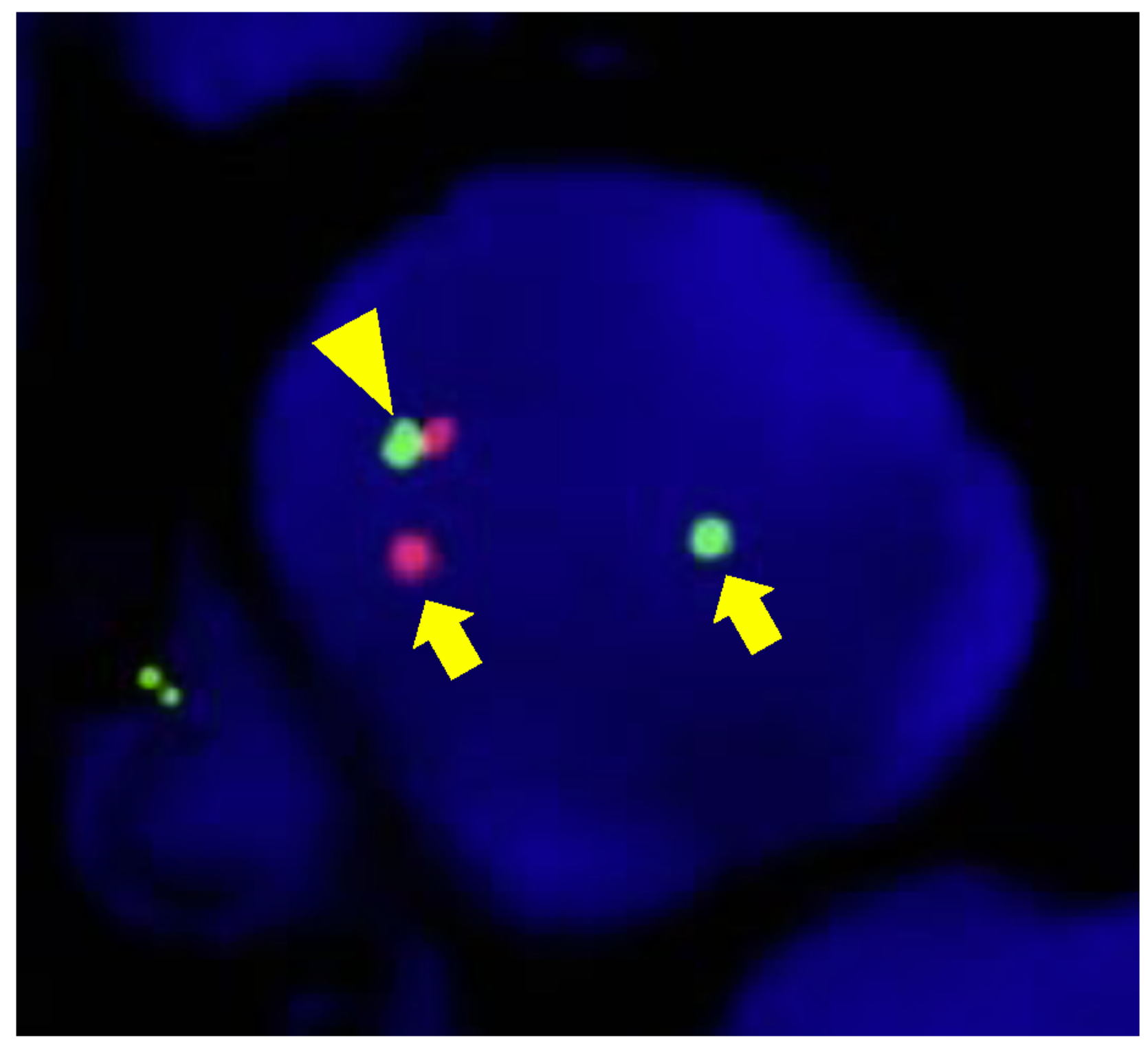

Figure 4

NUTM1 FISH of the tumor. Fluorescence in situ hybridization analysis (FISH) of NUTM1 showing a NUTM1 split signal in the tumor. Arrows indicate split signals, and an arrowhead indicates a fused signal. 

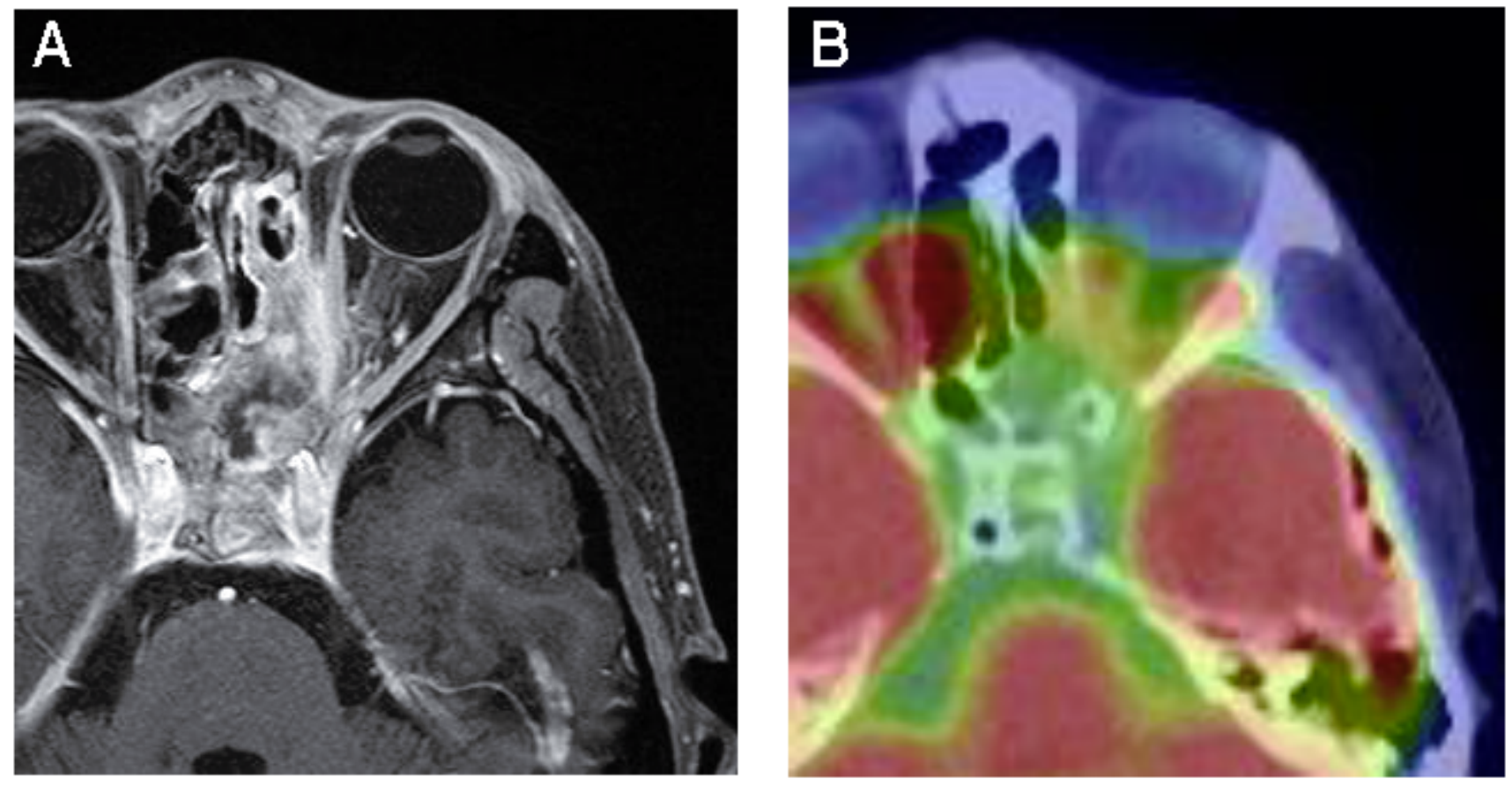

Figure 5

MRI (A) and PET-CT (B) after treatment. MRI, magnetic resonance imaging; PET, positron emission tomography, CT, computed tomography. MRI and PET-CT showed no abnormalities with signs of relapse.

\section{Supplementary Files}

This is a list of supplementary files associated with this preprint. Click to download.

- CAREguideline.pdf 